\title{
Asymptotics for incidence matrix classes
}

\author{
Peter Cameron, Thomas Prellberg and Dudley Stark
}

School of Mathematical Sciences

Queen Mary, University of London

Mile End Road, London, E1 4NS U.K.

$\{$ p.j.cameron, t.prellberg, d.s.stark\}@qmul.ac.uk

Submitted: Apr 4, 2006; Accepted: Oct 2, 2006; Published: Oct 12, 2006

Mathematics Subject Classifications: 05A16, 05C65

\begin{abstract}
We define incidence matrices to be zero-one matrices with no zero rows or columns. We are interested in counting incidence matrices with a given number of ones, irrespective of the number of rows or columns. A classification of incidence matrices is considered for which conditions of symmetry by transposition, having no repeated rows/columns, or identification by permutation of rows/columns are imposed. We find asymptotics and relationships for the number of matrices with $n$ ones in some of these classes as $n \rightarrow \infty$.
\end{abstract}

\section{Introduction}

In this paper we address the problem: How many zero-one matrices are there with exactly $n$ ones? Note that we do not specify in advance the number of rows or columns of the matrices. In order to make the answer finite, we assume that no row or column of such a matrix consists entirely of zeros. We call such a matrix an incidence matrix.

Rather than a single problem, there are many different problems here, depending on what symmetries and constraints are permitted. In general, we define $F_{i j k l}(n)$ to be the number of zero-one matrices with $n$ ones and no zero rows or columns, subject to the conditions

- $i=0$ if matrices differing only by a row permutation are identified, and $i=1$ if not;

- $j=0$ if matrices with two equal rows are forbidden, and $j=1$ if not;

- $k=0$ if matrices differing only by a column permutation are identified, and $k=1$ if not;

- $l=0$ if matrices with two equal columns are forbidden, and $l=1$ if not. 
The notation is chosen so that $F_{i j k l}(n)$ is a monotonic increasing function of each of the arguments $i, j, k, l$.

By transposition, it is clear that $F_{k l i j}(n)=F_{i j k l}(n)$ for all $i, j, k, l, n$. So, of the sixteen different functions defined above, only ten are distinct. However, among the problems with $k=i$ and $l=j$, we may decide that matrices which are transposes of each other are identified, leading to four further counting problems $\Phi_{i j}(n)$, for $i, j \in\{0,1\}$.

For example, there are four matrices with $n=2$, as shown:

$$
\left(\begin{array}{ll}
1 & 1
\end{array}\right), \quad\left(\begin{array}{l}
1 \\
1
\end{array}\right), \quad\left(\begin{array}{ll}
1 & 0 \\
0 & 1
\end{array}\right), \quad\left(\begin{array}{ll}
0 & 1 \\
1 & 0
\end{array}\right) .
$$

The first has repeated columns and the second has repeated rows. The third and fourth are equivalent under row permutations or column permutations, while the first and second are equivalent under transposition.

Table 1 gives some values of these functions. The values of $F_{1111}(n)$ are taken from the On-Line Encyclopedia of Integer Sequences [15], where this appears as sequence A101370 and $F_{0101}(n)$ appears as sequence A049311, while the values of $F_{0011}(n)$ and $F_{0111}(n)$ are obtained from a formula in Corollary 3.3 in [9] using MAPLE. Other computations were done with GAP [8].

Table 1: Some values of the fourteen functions

\begin{tabular}{||c||c|c|r|r|r|r|r|r|r||}
\hline$n$ & 1 & 2 & 3 & 4 & 5 & 6 & 7 & 8 & 9 \\
\hline$F_{0000}(n)$ & 1 & 1 & 2 & 4 & 7 & 16 & & & \\
$F_{0010}(n)$ & 1 & 1 & 3 & 11 & 40 & 174 & & & \\
$F_{1010}(n)$ & 1 & 2 & 10 & 72 & 624 & 6522 & & & \\
$F_{0001}(n)$ & 1 & 2 & 4 & 9 & 18 & 44 & & & \\
$F_{0011}(n)$ & 1 & 2 & 7 & 28 & 134 & 729 & 4408 & 29256 & 210710 \\
$F_{1001}(n)$ & 1 & 2 & 6 & 20 & 73 & 315 & & & \\
$F_{1011}(n)$ & 1 & 3 & 17 & 129 & 1227 & 14123 & & & \\
$F_{0101}(n)$ & 1 & 3 & 6 & 16 & 34 & 90 & 211 & 558 & 1430 \\
$F_{0111}(n)$ & 1 & 3 & 10 & 41 & 192 & 1025 & 6087 & 39754 & 282241 \\
$F_{1111}(n)$ & 1 & 4 & 24 & 196 & 2016 & 24976 & 361792 & 5997872 & 111969552 \\
$\Phi_{00}(n)$ & 1 & 1 & 2 & 3 & 5 & 11 & & & \\
$\Phi_{10}(n)$ & 1 & 2 & 8 & 44 & 340 & 3368 & & & \\
$\Phi_{01}(n)$ & 1 & 2 & 4 & 10 & 20 & 50 & & & \\
$\Phi_{11}(n)$ & 1 & 3 & 15 & 108 & 1045 & 12639 & 181553 & 3001997 & 55999767 \\
\hline
\end{tabular}

The counting problems can be re-interpreted in various ways:

Counting hypergraphs by weight: Given a hypergraph on the vertices $x_{1}, \ldots, x_{r}$, with edges $E_{1}, \ldots, E_{s}$ (each a non-empty set of vertices), the incidence matrix $A=\left(a_{i j}\right)$ is the matrix with $(i, j)$ entry 1 if $x_{i} \in E_{j}$, and 0 otherwise. The weight of the hypergraph is the sum of the cardinalities of the edges. Thus $F_{0101}(n)$ is the number of hypergraphs 
of weight $n$ with no isolated vertices, up to isomorphism; and $F_{1101}(n)$ is the number of (vertex)-labelled hypergraphs of weight $n$. Putting $k=1$ corresponds to labelling the edges, a less usual notion. Moreover, putting $l=0$ corresponds to counting simple hypergraphs (those without repeated edges). The condition $j=0$ is less natural in this respect, but corresponds to forbidding "repeated vertices" (pairs of vertices which lie in the same edges).

Counting bipartite graphs by edges: Given a zero-one matrix $A=\left(A_{i j}\right)$, there is a (simple) bipartite graph whose vertices are indexed by the rows and columns of $A$, with an edge from $r_{i}$ to $c_{j}$ if $A_{i j}=1$. The graph has a distinguished bipartite block (consisting of the rows). Thus, $F_{0101}(n)$ and $F_{1111}(n)$ count unlabelled and labelled bipartite graphs with $n$ edges and a distinguished bipartite block, respectively (where, in the labelled case, we assume that the labels of vertices in the distinguished bipartite block come first); $\Phi_{01}(n)$ counts unlabelled bipartite graphs with $n$ edges and a distinguished bipartition.

Counting pairs of partitions, or binary block designs: A block design is a set of plots carrying two partitions, the treatment partition and the block partition. It is said to be binary if no two distinct points lie in the same part of both partitions; that is, if the meet of the two partitions is the partition into singletons. Thus, $F_{0101}(n)$ is the number of binary block designs with $n$ plots. Putting $i=1$ or $k=1$ (or both) corresponds to labelling treatments or blocks (or both). Combinatorialists often forbid "repeated blocks" (this corresponds to putting $l=0$ ) although this is not natural from the point of view of experimental design. Similarly $j=0$ corresponds to forbidding "repeated treatments". The functions $\Phi_{i j}(n)$ count block designs up to duality (interchanging treatments and blocks), without or with treatment and block labelling and/or forbidding repeated blocks and treatments.

Counting orbits of certain permutation groups: A permutation group $G$ on a set $X$ is oligomorphic if the number $F_{n}^{*}(G)$ of orbits of $G$ on $X^{n}$ is finite for all $n$. Equivalently, the number $F_{n}(G)$ of orbits on ordered $n$-tuples of distinct elements is finite, and the number $f_{n}(G)$ of orbits on $n$-element subsets of $X$ is finite, for all $n$. These numbers satisfy various conditions, including the following:

- $F_{n}^{*}(G)=\sum_{k=1}^{n} S(n, k) F_{k}(G)$, where $S(n, k)$ are Stirling numbers of the second kind;

- $f_{n}(G) \leq F_{n}(G) \leq n ! f_{n}(G)$, where the right-hand bound is attained if and only if the group induced on a finite set by its setwise stabiliser is trivial.

For example, let $S$ be the symmetric group on an infinite set $X$, and $A$ the group of all order-preserving permutations of the rational numbers. Then $F_{n}(S)=f_{n}(S)=f_{n}(A)=1$ and $F_{n}(A)=n !$.

Now if $H$ and $K$ are permutation groups on sets $X$ and $Y$, then the direct product $H \times K$ acts coordinatewise on the Cartesian product $X \times Y$. It is easy to see that $F_{n}^{*}(H \times K)=F_{n}^{*}(H) F_{n}^{*}(K)$.

Let $\left(x_{1}, y_{1}\right), \ldots,\left(x_{n}, y_{n}\right)$ be $n$ distinct elements of $X \times Y$. If both $X$ and $Y$ are ordered, then the set of $n$ pairs can be described by a matrix with $n$ ones in these positions, where 
the rows and columns of the matrix are indexed by the sets $\left\{x_{1}, \ldots, x_{n}\right\}$ and $\left\{y_{1}, \ldots, y_{n}\right\}$ respectively (in the appropriate order). Moreover, if $X$ is not ordered, then we can represent the set of pairs as the equivalence class of this matrix under row permutations, and similarly for columns. Thus

$$
F_{0101}(n)=f_{n}(S \times S), \quad F_{1101}(n)=f_{n}(S \times A), \quad F_{1111}(n)=f_{n}(A \times A) .
$$

Moreover, the wreath product $H$ wr $C_{2}$ is the permutation group on $X^{2}$ generated by $H \times H$ together with the permutation $\tau:\left(x_{1}, x_{2}\right) \mapsto\left(x_{2}, x_{1}\right)$. The effect of $\tau$ is to transpose the matrix representing an orbit. So

$$
\Phi_{01}(n)=f_{n}\left(S \mathrm{wr} C_{2}\right), \quad \Phi_{11}(n)=f_{n}\left(A \mathrm{wr} C_{2}\right) .
$$

Discussion of this "product action" can be found in [5] and [13].

It is not clear how forbidding repeated rows or columns can be included in this interpretation.

\section{The asymptotics of $F_{1111}(n)$}

We will use both $F(n)$ and $F_{1111}(n)$ to denote the number of incidence matrices with $n$ ones. This is the largest of our fourteen functions, so its value gives an upper bound for all the others. Indeed, we will see later that $F_{i j k l}(n)=o\left(F_{1111}(n)\right)$ for $(i, j, k, l) \neq(1,1,1,1)$.

It is possible to compute this function explicitly. For fixed $n$, let $m_{i j}$ be the number of $i \times j$ matrices with $n$ ones (and no zero rows or columns). We set $m_{0,0}(0)=1$ and $F(0)=1$. Then

$$
\sum_{i \leq k} \sum_{j \leq l}\left(\begin{array}{l}
k \\
i
\end{array}\right)\left(\begin{array}{l}
l \\
j
\end{array}\right) m_{i j}=\left(\begin{array}{l}
k l \\
n
\end{array}\right)
$$

so by Möbius inversion,

$$
m_{k l}=\sum_{i \leq k} \sum_{j \leq l}(-1)^{k+l-i-j}\left(\begin{array}{l}
k \\
i
\end{array}\right)\left(\begin{array}{l}
l \\
j
\end{array}\right)\left(\begin{array}{l}
i j \\
n
\end{array}\right),
$$

and then

$$
F_{1111}(n)=\sum_{i \leq n} \sum_{j \leq n} m_{i j}
$$

For sequence $a_{n}, b_{n}$, we use the notation $a_{n} \sim b_{n}$ to mean $\lim _{n \rightarrow \infty} a_{n} / b_{n}=1$. It is clear from the argument above that

$$
F_{1111}(n) \leq\left(\begin{array}{c}
n^{2} \\
n
\end{array}\right) \sim \frac{1}{\sqrt{2 \pi n}}(n \mathrm{e})^{n},
$$

and of course considering permutation matrices shows that

$$
F_{1111}(n) \geq n ! \sim \sqrt{2 \pi n}\left(\frac{n}{\mathrm{e}}\right)^{n} .
$$




\section{Theorem 2.1}

$$
F_{1111}(n) \sim \frac{n !}{4} \mathrm{e}^{-\frac{1}{2}(\log 2)^{2}} \frac{1}{(\log 2)^{2 n+2}}
$$

We remark that for $n=10$, the asymptotic expression is about $2.5 \%$ less than the actual value of 2324081728 .

We have three different proofs of Theorem 2.1. One proof will be given in its entirety and the other two will be briefly sketched. Their full details can be found in [6]. We use the method of the first proof to bound $F_{1101}$ in Section 5. The ideas behind the third proof lead to a random algorithm for generating incidence matrices counted by $F_{1111}(n)$ and by $\Phi_{11}(n)$. The random algorithm provides an independent proof of the expression for $F_{1111}(n)$ used in the first proof.

First proof This proof uses a procedure which, when successful, generates an incidence matrix uniformly at random from all incidence matrices. The probability of success can be estimated and the asymptotic formula for $F_{1111}(n)$ results.

Let $R$ be a binary relation on a set $X$. We say $R$ is reflexive if $(x, x) \in R$ for all $x \in X$. We say $R$ is transitive if $(x, y) \in R$ and $(y, z) \in R$ implies $(x, z) \in R$. A partial preorder is a relation $R$ on $X$ which is reflexive and transitive. A relation $R$ is said to satisfy trichotomy if, for any $x, y \in X$, one of the cases $(x, y) \in R, x=y$, or $(y, x) \in R$ holds. We say that $R$ is a preorder if it is a partial preorder that satisfies trichotomy. The members of $X$ are said to be the elements of the preorder.

A relation $R$ is antisymmetric if, whenever $(x, y) \in R$ and $(y, x) \in R$ both hold, then $x=y$. A relation $R$ on $X$ is a partial order if it is reflexive, transitive, and antisymmetric. A relation is a total order, if it is a partial order which satisfies trichotomy. Given a partial preorder $R$ on $X$, define a new relation $S$ on $X$ by the rule that $(x, y) \in S$ if and only if both $(x, y)$ and $(y, x)$ belong to $R$. Then $S$ is an equivalence relation. Moreover, $R$ induces a partial order $\bar{R}$ on the set of equivalence classes of $S$ in a natural way: if $(x, y) \in R$, then $(\bar{x}, \bar{y}) \in \bar{R}$, where $\bar{x}$ is the $S$-equivalence class containing $x$ and similarly for $y$. We will call an $S$-equivalence class a block. If $R$ is a preorder, then the relation $\bar{R}$ on the equivalence classes of $S$ is a total order. See Section 3.8 and question 19 of Section 3.13 in [4] for more on the above definitions and results. Random preorders are considered in [7].

A preorder on $X$ with $k$ parts can also be described as a surjective mapping from $X$ to $\{1, \ldots, k\}$, where $(x, y) \in R$ if and only if $f(x) \leq f(y)$. The blocks are the sets $f^{-1}(i)$ for $i \in\{1, \ldots, k\}$.

The generating function and asymptotics of the number of preorders on $\{1, \ldots, n\}$ is given by Lovász [11, Exercise 1.15]. See also Section 3.8 and question 19 of Section 3.13 in [4] for more on the above definitions and results. Random preorders are considered in $[7]$. 
Given a preorder on elements $[n]:=\{1,2, \ldots, n\}$ with $K$ blocks, let $B_{1}, B_{2}, \ldots, B_{K}$ denote the blocks of the preorder. Generate two random preorders uniformly at random $B_{1}, B_{2}, \ldots, B_{K}$ and $B_{1}^{\prime}, B_{2}^{\prime}, \ldots, B_{L}^{\prime}$. For each $1 \leq i<j \leq n$, define the event $D_{i, j}$ to be

$$
D_{i, j}=\{\text { for each of the two preorders } i \text { and } j \text { are in the same block }\} .
$$

Furthermore, define

$$
W=\sum_{1 \leq i<j \leq n} I_{D_{i, j}},
$$

where the indicator random variables are defined by

$$
I_{D_{i, j}}= \begin{cases}1 & \text { if } D_{i, j} \text { occurs; } \\ 0 & \text { otherwise. }\end{cases}
$$

If $W=0$, then the procedure is successful, in which case $B_{k} \cap B_{l}^{\prime}$ consists of either 0 or 1 elements for each $1 \leq k \leq K$ and $1 \leq l \leq L$. If the procedure is successful, then we define the corresponding $K \times L$ incidence matrix $A$ by

$$
A_{k, l}= \begin{cases}1 & \text { if } B_{k} \cap B_{l}^{\prime} \neq \emptyset \\ 0 & \text { if } B_{k} \cap B_{l}^{\prime}=\emptyset\end{cases}
$$

It is easy to check that the above definition of $A$ in fact produces an incidence matrix and that each incidence matrix occurs in $n$ ! different ways by the construction. It follows that

$$
F_{1111}(n)=\frac{P(n)^{2} \mathbb{P}(W=0)}{n !},
$$

where $P(n)$ is the number of preorders on $n$ elements if $n \geq 1$ and $P(0)=1$.

It is known (see [1], for example) that the exponential generating function of $P(n)$ is

$$
\sum_{n=0}^{\infty} \frac{P(n)}{n !} z^{n}=\frac{1}{2-\mathrm{e}^{z}}
$$

The preceding equality implies that $P(n)$ has asymptotics given by

$$
P(n) \sim \frac{n !}{2}\left(\frac{1}{\log 2}\right)^{n+1},
$$

where, given sequences $a_{n}, b_{n}$ the notation $a_{n} \sim b_{n}$ means that $\lim _{n \rightarrow \infty} a_{n} / b_{n}=1$. It remains to find the asymptotics of $\mathbb{P}(W=0)$.

The $r$ th falling moment of $W$ is

$$
\begin{aligned}
\mathbb{E}(W)_{r} & =\mathbb{E} W(W-1) \cdots(W-r+1) \\
& =\mathbb{E}\left(\sum_{\text {pairs }\left(i_{s}, j_{s}\right) \text { different }} I_{i_{1}, j_{1}} \cdots I_{i_{r}, j_{r}}\right) \\
& =\mathbb{E}\left(\sum_{\text {all } i_{s} \text { and } j_{s} \text { different }} I_{i_{1}, j_{1}} \cdots I_{i_{r}, j_{r}}\right)+\mathbb{E}\left(\sum^{*} I_{i_{1}, j_{1}} \cdots I_{i_{r}, j_{r}}\right),
\end{aligned}
$$


with $\sum^{*}$ defined to be the sum with all pairs $\left(i_{s}, j_{s}\right)$ different, but not all $i_{s}, j_{s}$ different.

First we find the asymptotics of the first term in (7). For given sequences $i_{1}, i_{2}, \ldots, i_{r}$, $j_{1}, j_{2}, \ldots, j_{r}$, the expectation $\mathbb{E}\left(I_{i_{1}, j_{1}} \cdots I_{i_{r}, j_{r}}\right)$ is the number of ways of forming two preorders on the set of elements $[n] \backslash\left\{j_{1}, j_{2}, \ldots, j_{r}\right\}$ and then for each $s$ adding the element $j_{s}$ to the block containing $i_{s}$ in both preorders (which ensures that $D_{i_{s}, j_{s}}$ occurs for each $s$ ) and dividing the result by $P(n)^{2}$. Since the number of ways of choosing $i_{1}, i_{2}, \ldots, i_{r}$, $j_{1}, j_{2}, \ldots, j_{r}$ equals $\frac{n !}{2^{r}(n-2 r) !}$, This gives

$$
\begin{aligned}
\mathbb{E}\left(\sum_{\text {all } i_{s} \text { and } j_{s} \text { different }} I_{i_{1}, j_{1}} \cdots I_{i_{r}, j_{r}}\right) & =\frac{n !}{2^{r}(n-2 r) !} \frac{P(n-r)^{2}}{P(n)^{2}} \\
& \sim\left(\frac{(\log 2)^{2}}{2}\right)^{r},
\end{aligned}
$$

where we have used (5).

The second term is bounded in the following way. For each sequence $\left(i_{1}, j_{1}\right),\left(i_{2}, j_{2}\right), \ldots$, $\left(i_{s}, j_{s}\right)$ in the second term we form the graph $G$ on vertices $\bigcup_{s=1}^{r}\left\{i_{s}, j_{s}\right\}$ with edges $\bigcup_{s=1}^{r}\left\{\left\{i_{s}, j_{s}\right\}\right\}$. Consider the unlabelled graph $G^{\prime}$ corresponding to $G$ consisting of $v$ vertices and $c$ components. The number of ways of labelling $G^{\prime}$ to form $G$ is bounded by $n^{v}$. The number of preorders corresponding to this labelling is $P(n-v+c)$ because we form a preorder on $n-v+c$ vertices after which the vertices in the connected component of $G$ containing a particular vertex get added to that block. Therefore, we have

$$
\begin{aligned}
\mathbb{E}\left(\sum^{*} I_{i_{1}, j_{1}} \cdots I_{i_{r}, j_{r}}\right) & \leq \sum_{G^{\prime}} n^{v} \frac{P(n-v+c)^{2}}{P(n)^{2}} \\
& =\sum_{G^{\prime}} O\left(n^{2 c-v}\right)
\end{aligned}
$$

where the constant in $O\left(n^{2 c-v}\right)$ is uniform over all $G^{\prime}$ because $v \leq 2 r$. Since at least one vertex is adjacent to more than one edge, the graph $G$ is not a perfect matching. Furthermore, each component of $G$ contains at least two vertices. It follows that $2 c<v$ and, as a result,

$$
\mathbb{E}\left(\sum^{*} I_{i_{1}, j_{1}} \cdots I_{i_{r}, j_{r}}\right)=O\left(n^{-1}\right) .
$$

The preceding analysis shows that

$$
\mathbb{E}(W)_{r} \sim\left(\frac{(\log 2)^{2}}{2}\right)^{r}
$$

for each $r \geq 0$. The method of moments implies that the distribution converges weakly to the distribution of a Poisson $\left((\log 2)^{2} / 2\right)$ distributed random variable and therefore

$$
\mathbb{P}(W=0) \sim \exp \left(-\frac{(\log 2)^{2}}{2}\right) .
$$


Second proof (Sketch) First, the following expression for $F_{1111}(n)$ is given in terms of the number of preorders on $k$ elements as an alternating sum different from and simpler than (2):

$$
F_{1111}(n)=\frac{1}{n !} \sum_{k=1}^{n} s(n, k) P(k)^{2},
$$

where and $s(n, k)$ and $S(n, k)$ are Stirling numbers of the first and second kind respectively. As in the first proof, the number of pairs of preorders for which the meets of the blocks form a given $k$-partition of $[n]$ is $k ! F(k)$, so

$$
P(n)^{2}=\sum_{k=1}^{n} S(n, k) k ! F(k),
$$

and we obtain the result by inversion. Next, $P(k)$ is replaced by its asymptotic expression (5) with negligible error. Let

$$
F^{\prime}(n)=\frac{1}{4} \cdot \frac{1}{n !} \sum_{k=1}^{n} s(n, k)(k !)^{2} c^{k+1}
$$

where $c=1 /(\log 2)^{2}$ is as in the statement of the theorem. As we have argued, $F(n) \sim$ $F^{\prime}(n)$.

Now, $(-1)^{n-k} s(n, k)$ is the number of permutations in the symmetric group $S_{n}$ which have $k$ cycles. So we can write the formula for $F^{\prime}(n)$ as a sum over $S_{n}$, where the term corresponding to a permutation with $k$ cycles is $(-1)^{n-k}(k !)^{2} c^{k+1}$. In particular, the identity permutation gives us a contribution

$$
g(n)=\frac{1}{4} n ! c^{n+1}
$$

To show that $F^{\prime}(n) \sim C g(n)$ as $n \rightarrow \infty$, where $C=\exp \left(-(\log 2)^{2} / 2\right)$, we write $F^{\prime}(n)=$ $F_{1}^{\prime}(n)+F_{2}^{\prime}(n)+F_{3}^{\prime}(n)$, where the three terms are sums over the following permutations:

$F_{1}^{\prime}$ : all involutions (permutations with $\sigma^{2}=1$ );

$F_{2}^{\prime}$ : the remaining permutations with $k \geq\lceil n / 2\rceil ;$

$F_{3}^{\prime}$ : the rest of $S_{n}$.

A further argument shows that $F_{1}^{\prime}(n) \sim C g(n)$, while $F_{2}^{\prime}(n)=o(g(n))$ and $F_{3}^{\prime}(n)=$ $o(g(n))$.

Third proof (Sketch) If one is interested in asymptotic enumeration of $F(n)$, the formula (2), being a double sum over terms of alternating sign, is on first sight rather unsuitable for an asymptotic analysis. We present a derivation of the asymptotic form of $F(n)$ based on the following elegant and elementary identity. (This identity and equation (2) were also derived in [13].) 


\section{Proposition 2.2}

$$
F(n)=\sum_{k=0}^{\infty} \sum_{l=0}^{\infty} \frac{1}{2^{k+l+2}}\left(\begin{array}{l}
k l \\
n
\end{array}\right) .
$$

Proof Insert

$$
1=\sum_{k=i}^{\infty} \frac{1}{2^{k+1}}\left(\begin{array}{l}
k \\
i
\end{array}\right)=\sum_{l=j}^{\infty} \frac{1}{2^{l+1}}\left(\begin{array}{l}
l \\
j
\end{array}\right)
$$

into (3) and resum using (1).

The sum in (9) is dominated by terms where $k l \gg n$. In this regime, using

$$
\left(\begin{array}{c}
k l \\
n
\end{array}\right) \sim \frac{(k l)^{n}}{n !} \mathrm{e}^{-\frac{n^{2}}{2 k l}}
$$

and approximating the sum in (9) by an integral (cf. Euler-Maclaurin) leads to

$$
\begin{aligned}
F(n) & \sim \frac{1}{4 n !} \int \mathrm{d} k \int \mathrm{d} l \frac{(k l)^{n}}{2^{k+l}} \mathrm{e}^{-\frac{n^{2}}{2 k l}} \\
& =\frac{n^{2 n+2}}{4 n !} \int \mathrm{d} \kappa \int \mathrm{d} \lambda \mathrm{e}^{n(\log \kappa-\kappa \log 2)} \mathrm{e}^{n(\log \lambda-\lambda \log 2)} \mathrm{e}^{-\frac{1}{2 \kappa \lambda}}
\end{aligned}
$$

For $n$ large, the integrals are dominated by a small neighborhood around their respective saddles. As $\mathrm{e}^{-\frac{1}{2 \kappa \lambda}}$ is independent of $n$, we can treat the integrals separately. Using $w(\kappa)=\log \kappa-\kappa \log 2$, the saddle $\kappa_{s}=\frac{1}{\log 2}$ is determined from $w^{\prime}\left(\kappa_{s}\right)=0\left(\lambda_{s}=\frac{1}{\log 2}\right.$ analogously). Approximating the integrals by a Gaussian around the saddle point gives

$$
\begin{aligned}
F(n) & \sim \frac{n^{2 n+2}}{4 n !} \mathrm{e}^{n w\left(\kappa_{s}\right)} \sqrt{\frac{2 \pi}{n\left|w^{\prime \prime}\left(\kappa_{s}\right)\right|}} \mathrm{e}^{n w\left(\lambda_{s}\right)} \sqrt{\frac{2 \pi}{n\left|w^{\prime \prime}\left(\lambda_{s}\right)\right|}} \mathrm{e}^{-\frac{1}{2 \kappa_{s} \lambda_{s}}} \\
& =\frac{n^{2 n+2}}{4 n !}\left(\mathrm{e}^{n(\log \log 2-1)} \sqrt{\frac{2 \pi}{n(\log 2)^{2}}}\right)^{2} \mathrm{e}^{-\frac{1}{2}(\log 2)^{2}}
\end{aligned}
$$

which simplifies to the desired result.

\section{Generating random incidence matrices}

It is easily shown that (4) implies that

$$
P(n)=\sum_{k=0}^{\infty} \frac{k^{n}}{2^{k+1}} .
$$

Hence, the distribution $\pi_{k}$ on the natural numbers defined by

$$
\pi_{k}=\frac{k^{n}}{P(n) 2^{k+1}}
$$

is a probability distribution. The following way of generating preorders uniformly at random was given in [12]. 
Theorem 3.1 (Maassen, Bezembinder) Let $A$ be a set of $n$ elements, $n \geq 1$. Let a random preorder $R$ be generated by the following algorithm:

(i) Draw an integer-valued random variable $K$ according to the probability distribution $\pi_{k}$.

(ii) To each $a \in A$ assign a random score $X_{a}$ according to the uniform distribution on $\{1,2, \ldots, K\}$.

(iii) Put $(a, b) \in R$ if and only if $X_{a} \leq X_{b}$.

Then all of the $P(n)$ possible preorders on $A$ are obtained with the same probability $1 / P(n)$.

A referee remarked that a defect of this algorithm is the need to know $P(n)$ in advance in order to calculate the probability distribution in Step (i), and suggested that there might be a Metropolis-type Markov chain whose limiting distribution is uniform. The same comment applies to our algorithm below for a random incidence matrix. This would be desirable if one is interested in practical applications.

Incidence matrices counted by $F_{1111}(n)$ can be generated uniformly at random by a similar algorithm. Define a integer valued joint probability distribution function $\rho_{k, l}$ by

$$
\rho_{k, l}=\frac{1}{F_{1111}(n)}\left(\begin{array}{c}
k l \\
n
\end{array}\right) 2^{-k-l-2} .
$$

Theorem 3.2 The following algorithm generates a random incidence matrix counted by $F_{1111}(n)$.

(i) Draw integer-valued random variables $K$ and $L$ according to the joint probability distribution $\rho_{k, l}$.

(ii) Choose a 0-1 matrix with $K$ rows, $L$ columns, $n$ 1's and $K L-n$ 0's uniformly at random.

(iii) Delete all rows and columns for which all entries are 0.

Proof Denote a 0-1 matrix with $k$ rows, $l$ columns, and $n$ 1's a $(k, l)$-matrix. Denote an incidence matrix with $i$ rows, $j$ columns, and $n$ 1's a $(i, j)$-incidence matrix. Now, every $(k, l)$-matrix is generated with equal probability

$$
\frac{\rho_{k, l}}{\left(\begin{array}{c}
k l \\
n
\end{array}\right)}=\frac{2^{-k-l-2}}{F_{1111}(n)}
$$

and every $(i, j)$-incidence matrix is generated from $\left(\begin{array}{l}k \\ i\end{array}\right)\left(\begin{array}{l}l \\ j\end{array}\right)(k, l)$-matrices. Averaging over the probability distribution, it follows that every $(i, j)$-incidence matrix is generated with probability

$$
p(i, j)=\left(\begin{array}{l}
k l \\
n
\end{array}\right)^{-1} \sum_{k, l}\left(\begin{array}{l}
k \\
i
\end{array}\right)\left(\begin{array}{l}
l \\
j
\end{array}\right) \rho_{k, l}
$$

Using (10), this sum simplifies to $p(i, j)=1 / F_{1111}(n)$. 


\section{Counting symmetric matrices}

In this section we find the asymptotics for $\Phi_{11}(n)$ and show:

Proposition 4.1 $\Phi_{11}(n) \sim \frac{1}{2} F_{1111}(n)$.

Proof Clearly we have $\Phi_{11}(n)=\frac{1}{2}\left(F_{1111}(n)+S_{11}(n)\right)$, where $S_{11}(n)$ is the number of symmetric matrices with $n$ ones having no zero rows or columns, where repeated rows or columns are allowed and row or column permutations are not permitted. So it suffices to show that $S_{11}(n)=o\left(F_{1111}(n)\right)$.

Now let $I(n)$ be the number of solutions of $\sigma^{2}=1$ in the symmetric group $S_{n}$. Then we have

$$
I(n) \leq S_{11}(n) \leq I(n) P(n) / n !
$$

The lower bound is clear by considering symmetric permutation matrices. For the upper bound, our analysis of $F_{1111}(n)$ shows that $n ! S_{11}(n)$ is the number of pairs $\left(R_{1}, R_{2}\right)$ of preorders on $\{1, \ldots, n\}$ such that no two points $i$ and $j$ lie in the same block for both preorders, and additionally such that $R_{1}$ and $R_{2}$ are interchanged by some involution $\sigma$ of $\{1, \ldots, n\}$ (corresponding to transposition of the matrix). So instead of choosing $R_{1}$ and $R_{2}$, we can choose $R_{1}$ and $\sigma$ and let $R_{2}=R_{1}^{\sigma}$; there are $P(n) I(n)$ choices, and this is an overcount because of the extra condition that must hold on $\left(R_{1}, R_{2}\right)$.

Now $I(n)$ is just a little larger than $\sqrt{n !}$ : in fact,

$$
I(n) \sim \frac{n^{n / 2}}{\sqrt{2} \mathrm{e}^{n / 2-\sqrt{n}+1 / 4}}
$$

(see [3, p. 347]). We have seen that $P(n) / n ! \sim A(1 / \log 2)^{n}$. So the conclusion follows from Theorem 2.1.

It is possible to show that the upper bound for $S_{11}(n)$ is correct, apart from a constant factor:

Proposition $4.2 S_{11}(n) \sim C_{s} \cdot I(n) P(n) / n$ !, where $C_{s}=\frac{1}{2} \mathrm{e}^{-(\log 2)^{2} / 4} \approx 0.44341$. In other words, if we choose randomly a preorder $R$ and an involution $\sigma \in S_{n}$, the probability that no two points lie in the same part in both $R$ and $R^{\sigma}$ tends to $C_{s}$ as $n \rightarrow \infty$.

Proof Let $\mu_{i}=\mu_{i}(n)$ be the number of $i \times i$ symmetric incidence matrices with $n$ ones. Let $s_{k}$ be the number of $k \times k$ symmetric matrices with $n$ ones, given by

$$
s_{k}=\sum_{j=0}^{\lfloor n / 2\rfloor}\left(\begin{array}{c}
k \\
2 \\
j
\end{array}\right)\left(\begin{array}{c}
k \\
n-2 j
\end{array}\right),
$$

where $j$ represents the number of ones off of the diagonal. Then

$$
s_{k}=\sum_{i=1}^{k}\left(\begin{array}{l}
k \\
i
\end{array}\right) \mu_{i}
$$

THE ELECTRONIC JOURNAL OF COMBINATORICS 13 (2006), \#R85 
and

$$
S_{11}(n)=\sum_{i=1}^{n} \mu_{i}=\sum_{k=1}^{\infty} \frac{s_{k}}{2^{k+1}}
$$

by (10), leading to

$$
S_{11}(n)=\sum_{k=0}^{\infty} \frac{1}{2^{k+1}} \sum_{j=0}^{\infty}\left(\begin{array}{c}
\left(\begin{array}{c}
k \\
2
\end{array}\right) \\
j
\end{array}\right)\left(\begin{array}{c}
k \\
n-2 j
\end{array}\right) .
$$

To compute this sum asymptotically, we approximate for $m \gg l \gg 1$

$$
\left(\begin{array}{c}
m \\
l
\end{array}\right) \sim \frac{m^{l}}{l !} \mathrm{e}^{-l^{2} / 2 m} .
$$

The sums are dominated near $k \approx n / \log 2$ and $j \approx(n-\sqrt{n}) / 2$, so that we can justify replacing the binomial coefficients by this approximation. We get

$$
\begin{aligned}
S_{11}(n) & \sim \sum_{k=0}^{\infty} \frac{1}{2^{k+1}} \sum_{j=0}^{\infty} \frac{\left(\begin{array}{l}
k \\
2
\end{array}\right)^{j}}{j !} \frac{k^{n-2 j}}{(n-2 j) !} \mathrm{e}^{-j^{2} / 2\left(\begin{array}{c}
k \\
2
\end{array}\right)-(n-2 j)^{2} / 2 k} \\
& \sim \sum_{k=0}^{\infty} \frac{k^{n}}{2^{k+1}} \sum_{j=0}^{\infty} \frac{1}{j !(n-2 j) ! 2^{j}} \mathrm{e}^{-j / k-j^{2} / k^{2}-(n-2 j)^{2} / 2 k}
\end{aligned}
$$

where in the last step we also replaced $(1-1 / k)^{j} \sim \mathrm{e}^{-j / k}$. Due to the concentration of the sum near $k \approx n / \log 2$ and $j \approx(n-\sqrt{n}) / 2$, the argument of the exponential can be replaced by

$$
\mathrm{e}^{-j / k-j^{2} / k^{2}-(n-2 j)^{2} / 2 k} \sim \mathrm{e}^{-\log 2-(\log 2 / 2)^{2}}=\frac{1}{2} \mathrm{e}^{-(\log 2)^{2} / 4}=C_{s},
$$

where $C_{s}$ is as in the Proposition. Identifying

$$
P(n)=\sum_{k=0}^{\infty} \frac{k^{n}}{2^{k+1}}
$$

and

$$
I(n)=\sum_{j=0}^{\infty} \frac{n !}{j !(n-2 j) ! 2^{j}}
$$

we arrive at

$$
S_{11}(n) \sim C_{s} \cdot I(n) P(n) / n !
$$

One may also generate matrices from $S_{11}$ uniformly at random.

Define an integer valued probability distribution function $\psi_{k}$ by

$$
\psi_{k}=\frac{s_{k} 2^{-k-1}}{S_{11}(n)} .
$$


Theorem 4.3 The following algorithm generates a random incidence matrix counted by $S_{11}(n)$.

(i) Draw integer-valued random variables $K$ according to the probability distribution $\psi_{k}$.

(ii) Choose a $K \times K$ symmetric zero-one matrix with $n$ ones and $K^{2}-n$ zeros uniformly at random.

(iii) Delete all rows and columns for which all entries are zero.

The proof of Theorem 4.3 is similar to the proof of Theorem 3.2.

In general, we have $\Phi_{i j}(n)=\frac{1}{2}\left(F_{i j i j}(n)+S_{i j}(n)\right)$, where

- if $i=1$, then $S_{i j}(n)$ is the number of symmetric matrices with $n$ ones and no zero rows, where repeated rows are forbidden if $j=0$ and permitted if $j=1$;

- if $i=0$, then $S_{i j}(n)$ is the number of classes of matrices with $n$ ones and no zero rows (up to row and column permutations) which are closed under transposition, with the same interpretation of $j$ as in the other case.

We do not yet have asymptotics for these. It seems likely that, in all four cases, $S_{i j}(n)=o\left(F_{i j i j}(n)\right)$, so that $\Phi_{i j}(n) \sim \frac{1}{2} F_{i j i j}(n)$. Table 2 gives some values of these functions.

Table 2: Some counts for symmetric matrices and classes

\begin{tabular}{||c||c|c|c|r|r|r|r|r|r|r||}
\hline$n$ & 1 & 2 & 3 & 4 & 5 & 6 & 7 & 8 & 9 & 10 \\
\hline$S_{00}(n)$ & 1 & 1 & 2 & 2 & 3 & 6 & & & & \\
$S_{01}(n)$ & 1 & 1 & 2 & 4 & 6 & 10 & & & & \\
$S_{10}(n)$ & 1 & 2 & 6 & 16 & 56 & 214 & 866 & 3796 & 17468 & \\
$S_{11}(n)$ & 1 & 2 & 6 & 20 & 74 & 302 & 1314 & 6122 & 29982 & 154718 \\
\hline
\end{tabular}

\section{The function $F_{1011}(n)$}

Recall that the number of incidence matrices with $n$ ones, no repeated rows and matrices equal by row or column permutations unidentified is denoted by $F_{1011}(n)$. In this section we will show

Theorem 5.1 We have

$$
F_{1011}(n)=o\left(F_{1111}(n)\right)
$$


Proof We will use the probabilistic method and the notation used in the proof of Theorem 2.1. The idea behind the proof is to show that the probability tends to 0 that a randomly chosen incidence matrix counted by $F_{1111}(n)$ does not have two rows with each containing all zeroes except for a single one in the same column.

Define $E_{i, j}, 1 \leq i, j \leq n$, to be the event that both $\{i\}$ and $\{j\}$ are blocks in the first preorder and that $i$ and $j$ belong to the same block of the second preorder. When $W=0$, $E_{i, j}$ corresponds to the event that the rows corresponding to the blocks containing $i$ and $j$ in the incidence matrix are different and contain unique ones appearing in the same column.

Let $P(n, k)$ be the number of preorders on $n$ elements with $k$ blocks. Given a power series $f(z)=\sum_{n=0}^{\infty} f_{n} z^{n}$, define $\left[z^{n}\right] f(z)=f_{n}$. We find that for any $1 \leq i<j \leq n$,

$$
\begin{aligned}
\mathbb{P}\left(E_{i, j}\right) & =\sum_{k=1}^{n-2} \frac{P(n-2, k)}{P(n)}(k+2)(k+1) \cdot \frac{P(n-1)}{P(n)} \\
& =\frac{P(n-1)}{P(n)^{2}} \sum_{k=1}^{n-2}(k+2)(k+1) P(n-2, k)
\end{aligned}
$$

Using Lemma 1.1 of [7], we find that

$$
\begin{aligned}
\sum_{k=1}^{n-2}(k+2)(k+1) P(n-2, k) & =(n-2) !\left[z^{n-2}\right]\left(\sum_{n=0}^{\infty}(n+2)(n+1)\left(\mathrm{e}^{z}-1\right)^{n}\right) \\
& =(n-2) !\left[z^{n-2}\right] \frac{d^{2}}{d u^{2}}\left(\left.\frac{u^{2}}{1-u}\right|_{u=\mathrm{e}^{z}-1}\right) .
\end{aligned}
$$

When singularity analysis (see Section 11 of [14]) can be applied, as in this case, the asymptotics of the coefficients of a generating function are determined by the degree of its pole of smallest modulus. Therefore,

$$
\sum_{k=1}^{n-2}(k+2)(k+1) P(n-2, k) \sim(n-2) !\left[z^{n-2}\right]\left(\frac{2\left(\mathrm{e}^{z}-1\right)^{2}}{\left(2-\mathrm{e}^{z}\right)^{3}}\right) .
$$

The singularity of smallest modulus of $\left(2-\mathrm{e}^{z}\right)^{-1}$ occurs at $z=\log 2$ with residue

$$
\lim _{z \rightarrow \log 2}\left(\frac{z-\log 2}{2-\mathrm{e}^{z}}\right)=\lim _{z \rightarrow \log 2}\left(\frac{1}{-\mathrm{e}^{z}}\right)=-\frac{1}{2},
$$

by l'Hôpital's rule. Hence,

$$
\sum_{k=1}^{n-2}(k+2)(k+1) P(n-2, k) \sim \frac{(n-2) !}{4}\left[z^{n-2}\right](\log 2-z)^{-3}
$$

from which singularity analysis and (5) give

$$
\sum_{k=1}^{n-2}(k+2)(k+1) P(n-2, k) \sim \frac{(n-2) !}{8}(\log 2)^{-n-1} n^{2} .
$$


The result of using (13) in (12) is

$$
\mathbb{P}\left(E_{i, j}\right) \sim \frac{\log 2}{4 n} .
$$

Define $X$ to be

$$
X=\sum_{1 \leq i<j \leq n} I_{E_{i, j}}
$$

so that, conditional on the event $\{W=0\}$, the event $\{X>0\}$ implies that the incidence matrix produced by the algorithm has repeated rows. The expectation of $X$ is

$$
\mathbb{E}(X)=\left(\begin{array}{l}
n \\
2
\end{array}\right) \mathbb{P}\left(E_{i, j}\right) \sim \frac{\log 2}{8} n
$$

We will next show that

$$
\mathbb{E}\left((W)_{r} \cap E_{i, j}\right) \sim\left(\frac{(\log 2)^{2}}{2}\right)^{r} \frac{\log 2}{4 n} .
$$

The analog of (6) is

$$
\begin{aligned}
\mathbb{E}\left((W)_{r} \cap E_{i, j}\right)= & \mathbb{E}\left(\sum_{\substack{\text { pairs } \\
\left.\text { pis, } i_{s}\right) \text { different } \\
i_{s} \neq i, j_{s} \neq j}} I_{i_{1}, j_{1}} \cdots I_{i_{r}, j_{r}} I_{E_{i, j}}\right) \\
= & \mathbb{E}\left(\sum_{\substack{\text { all } \\
i_{s} \text { and } j_{s} \text { different } \\
i_{s} \neq i, j_{s} \neq j}} I_{i_{1}, j_{1}} \cdots I_{i_{r}, j_{r}} I_{E_{i, j}}\right) \\
& +\mathbb{E}\left(\sum^{* * *} I_{i_{1}, j_{1}} \cdots I_{i_{r}, j_{r}} I_{E_{i, j}}\right)
\end{aligned}
$$

with $\sum^{* *}$ defined to be the sum with all pairs $\left(i_{s}, j_{s}\right)$ different, but not all $i_{s}, j_{s}$ different. The first term corresponds to two preorders formed in the following way. The $i_{s}, j_{s}$ are first selected. One preorder is formed from the set of elements $[n] \backslash\left\{i, j, j_{1}, j_{2}, \ldots, j_{r}\right\}$, the element $j_{s}$ is added to the block containing $i_{s}$ for each $s$, and then blocks $\{i\}$ and $\{j\}$ are inserted in the preorder. Another preorder is formed from the set of elements $[n] \backslash\left\{j, j_{1}, j_{2}, \ldots, j_{r}\right\}$, the element $j_{s}$ is added to the block containing $i_{s}$ for each $s$, and then the element $j$ is added to the block containing $i$. As a result,

$$
\begin{aligned}
& \mathbb{E}\left(\sum_{\substack{\text { pairs } \\
\text { piss,js) } \\
i_{s} \neq i, j_{s} \neq j}} I_{i_{1}, j_{1}} \cdots I_{i_{r}, j_{r}}\right) \\
= & \frac{(n-2) !}{2^{r}(n-2-2 r) !} \sum_{k=1}^{n-2-r} \frac{P(n-2-r, k)}{P(n)}(k+2)(k+1) \frac{P(n-1-r)}{P(n)} \\
\sim & \left(\frac{(\log 2)^{2}}{2}\right)^{r} \frac{\log 2}{4 n}
\end{aligned}
$$


where we have used (5) and the asymptotic form (13).

The second term is bounded using the same method that was used to bound $\sum^{*}$ in the proof of Theorem 2.1. Letting $G^{\prime \prime}$ be an index over graphs on $n$ vertices with two labelled disconnected vertices $i$ and $j$ and $n-2$ unlabelled vertices which is not a matching on the unlabelled vertices, we have

$$
\begin{aligned}
& \mathbb{E}\left(\sum^{* *} I_{i_{1}, j_{1}} \cdots I_{i_{r}, j_{r}} I_{E_{i, j}}\right) \\
\leq & \sum_{G^{\prime \prime}}(n-2)^{v} \sum_{k} \frac{P(n-2-v+c, k)}{P(n)}(k+1)(k+2) \frac{P(n-1-v+c)}{P(n)} \\
= & \sum_{G^{\prime \prime}} O\left(n^{2 c-v-1}\right) \\
= & O\left(n^{-2}\right) .
\end{aligned}
$$

Consequently we have shown (16).

The asymptotics (14) and (16) and method of moments argument giving (8) imply that

$$
\mathbb{P}\left(W=0 \mid E_{i, j}\right) \sim \exp \left(-\frac{(\log 2)^{2}}{2}\right)
$$

and therefore an application of Bayes' Theorem with (8) and (14) results in

$$
\mathbb{P}\left(E_{i, j} \mid W=0\right) \sim \frac{\log 2}{4 n} .
$$

The observations above result in

$$
\mathbb{E}(X \mid W=0) \sim \frac{\log 2}{8} n .
$$

Comparison of (15) and (17) makes it clear that that conditioning on the event $\{W=0\}$ does not asymptotically affect the expectation of $X$.

In a similar way we can find the asymptotics of the conditional second falling moment $\mathbb{E}(X(X-1) \mid Y=0)$. The unconditioned second moment equals

$$
\begin{aligned}
\mathbb{E}(X(X-1))= & \sum_{\left(i_{1}, j_{1}\right) \neq\left(i_{2}, j_{2}\right)} \mathbb{P}\left(E_{i_{1}, j_{1}} \cap E_{i_{2}, j_{2}}\right) \\
= & \sum_{\left\{i_{1}, j_{1}\right\} \cap\left\{i_{2}, j_{2}\right\}=\emptyset} \mathbb{P}\left(E_{i_{1}, j_{1}} \cap E_{i_{2}, j_{2}}\right)+\sum_{\left|\left\{i_{1}, j_{1}\right\} \cap\left\{i_{2}, j_{2}\right\}\right|=1} \mathbb{P}\left(E_{i_{1}, j_{1}} \cap E_{i_{2}, j_{2}}\right) \\
= & \frac{n !}{4(n-4) !} \sum_{k=1}^{n-4} \frac{P(n-4, k)}{P(n)}(k+1)(k+2)(k+3)(k+4) \frac{P(n-2)}{P(n)} \\
& +O\left(n^{3} \sum_{k=1}^{n-4} \frac{P(n-3, k)}{P(n)}(k+1)(k+2)(k+3) \frac{P(n-2)}{P(n)}\right)
\end{aligned}
$$


An application of singularity analysis as used to derive (13) produces

$$
\mathbb{E}(X(X-1))=\frac{(\log 2)^{2}}{64} n^{2}+O(n) .
$$

Arguing as we did for $\mathbb{E}(X \mid W=0)$ shows that

$$
\mathbb{E}(X(X-1) \mid W=0)=\frac{(\log 2)^{2}}{64} n^{2}+O(n) ;
$$

we omit the details.

The variance of $X$ conditioned on $W=0$ is

$$
\begin{aligned}
\operatorname{Var}(X \mid W=0) & =\mathbb{E}(X(X-1) \mid W=0)+\mathbb{E}(X \mid W=0)-(\mathbb{E}(X \mid W=0))^{2} \\
& =o\left(n^{2}\right) .
\end{aligned}
$$

Chebyshev's inequality applied with (17) and (18) now gives

$$
\mathbb{P}(X=0 \mid W=0)=o(1) .
$$

Hence, an asymptotically insignificant fraction of incidence matrices do not have repeated rows which implies that $F_{1011}(n)=o\left(F_{1111}(n)\right)$.

\section{The functions $F_{0011}(n)$ and $F_{0111}(n)$}

The function $F_{0111}(n)$ counts vertex-labelled hypergraphs on $n$ vertices, while $F_{0011}(n)$ counts the simple vertex-labelled hypergraphs. For completeness, we include the formulae from the work of Martin Klazar [9].

Theorem 6.1 (a) For all n, we have

$$
\begin{aligned}
& \left.F_{0011}(n)=\sum_{\lambda \vdash n} \sum_{j=l}^{n} \prod_{i=1}^{l}\left(\begin{array}{c}
j \\
i
\end{array}\right)\right) \sum_{m=j}^{n}(-1)^{m-j}\left(\begin{array}{c}
m \\
j
\end{array}\right),
\end{aligned}
$$

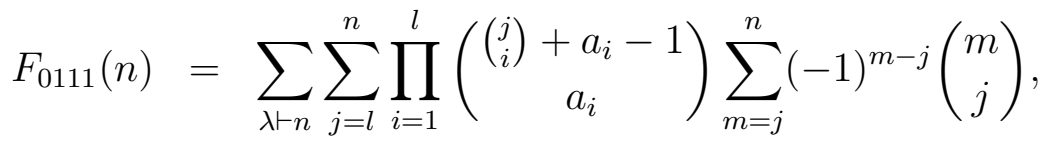

where $\lambda=1^{a_{1}} 2^{a_{2}} \ldots l^{a_{l}}$ is a partition of $n$ with $a_{l}>0$.

(b) For all n, we have

$$
F_{0011}(n) \leq F_{0111}(n) \leq 2 F_{0011}(n) .
$$

Part (b) raises the question of whether $F_{0011}(n) / F_{0111}(n)$ tends to a limit as $n \rightarrow \infty$, and particular, whether the limit is 1 (that is, whether almost all labelled hypergraphs are simple). 
The paper [9] also gives recurrence relations for the two functions. Klazar subsequently showed [10] that both functions are asymptotically

$$
(1 / \log 2+o(1))^{n} b(n),
$$

where $b(n)$ is the $n$th Bell number (the number of partitions of $\{1, \ldots, n\}$ ). Details of the asymptotics of $b(n)$ can be found in [14]. In particular, since $b(n) / n !=(1+o(1))^{n}$, we see that $F_{0111}(n)=o\left(F_{1111}(n)\right)$, and in fact

$$
F_{0111}(n)=(\log 2+o(1))^{n} F_{1111}(n) .
$$

This and the result of the last section, together with the facts that $F_{1101}(n)=F_{0111}(n)$ and $F_{1110}(n)=F_{1011}(n)$ and that $F_{i j k l}(n)$ is monotone increasing in each of $i, j, k, l$, justify our earlier claim that $F_{i j k l}(n)=o\left(F_{1111}(n)\right)$ for $(i, j, k, l) \neq(1,1,1,1)$.

\section{A rough lower bound for $F_{0001}(n)$}

The number $F_{0101}(n)$ of unlabelled hypergraphs with weight $n$ is not smaller than the number of graphs with $n / 2$ edges and no isolated vertices. We show that this number grows faster than exponentially. In fact, our argument applies to $F_{0001}$, since we use simple graphs.

Consider simple graphs with $m$ vertices and $n$ edges, where $m=o(n)$ and $n=o\left(m^{2}\right)$. The number of such graphs, up to isomorphism, is at least

$$
\frac{\left(\begin{array}{c}
m(m-1) / 2 \\
n
\end{array}\right)}{m !}>\frac{\left(c m^{2}\right)^{n}}{n ! m !}>\frac{c^{n} m^{2 n}}{n^{n} m^{m}}
$$

for some constant $c$. Put $F$ equal to the logarithm of the right-hand side:

$$
F=c^{\prime} n+2 n \log m-n \log n-m \log m
$$

for some constant $c^{\prime}$. Putting $m=c^{\prime \prime} n / \log n$, for some constant $c^{\prime \prime}$, we get

$$
F=n \log n-2 n \log \log n+O(n) .
$$

We conclude:

Proposition 7.1 For any $\epsilon>0$, we have

$$
F_{0001}(n) \geq\left(\frac{n}{(\log n)^{2+\epsilon}}\right)^{n}
$$

for $n \geq n_{0}(\epsilon)$.

Remark The asymptotics of the number of graphs with no isolated vertices, having a given number of vertices and edges, has a long history: see [16] for an early paper on this topic, and [2] for a recent result. 


\section{References}

[1] J. P. Barthelemy, An asymptotic equivalent for the number of total preorders on a finite set, Discrete Math. 29 (1980) 311-313.

[2] E. A. Bender, E. R. Canfield and B. D. McKay, The asymptotic number of labeled graphs with $n$ vertices, $q$ edges, and no isolated vertices, J. Combinatorial Theory (A) 80 (1997), 124-150.

[3] E. A. Bender and S. G. Williamson, Foundations of Combinatorics with Applications, http://math.ucsd.edu/ ebender/CombText/

[4] P. J. Cameron, Combinatorics: Topics, Techniques, Algorithms, Cambridge University Press, 1994.

[5] P. J. Cameron, D. A. Gewurz and F. Merola, Product action, to appear.

[6] P. J. Cameron, Thomas Prellberg and Dudley Stark, Asymptotic enumeration of incidence matrices, Journal of Physics (Conference Series), to appear.

[7] P. J. Cameron and Dudley Stark, Random preorders, submitted.

[8] The GAP Group, GAP — Groups, Algorithms, and Programming, Version 4.3, 2002, http://www.gap-system.org

[9] M. Klazar, Extremal problems for ordered hypergraphs: small patterns and some enumeration, Discrete Appl. Math. 143 (2004), 144-154.

[10] M. Klazar, Counting set systems by weight, Electr. J. Combinatorics 12(1) (2005), \#R11, (8pp).

[11] L. Lovász, Combinatorial Problems and Exercises, North-Holland, Amsterdam, 1978.

[12] H. Maassen and T. Bezembinder, Generating random weak orders and the probability of a Condorcet winner. Soc. Choice Welfare 19 (2002) 517-532.

[13] M. Maia and M. Mendez, On the arithmetic product of combinatorial species, preprint available from arXiv:math.C0/0503436

[14] A. M. Odlyzko, Asymptotic enumeration methods, In R. L. Graham, M. Grötschel and L. Lovász (eds.), Handbook of Combinatorics, Vol. 2, North-Holland, Amsterdam, 1995, pp. 1063-1229.

[15] N. J. A. Sloane (ed.), The On-Line Encyclopedia of Integer Sequences, http://www.research.att.com/ njas/sequences/

[16] E. M. Wright, Two problems in the enumeration of unlabelled graphs. Discrete Math. 9 (1974), 289-292. 